\title{
MEDIDAS VOCAIS ACÚSTICAS NA DOENÇA DE PARKINSON: ESTUDO DE CASOS
}

\section{Vocal acoustic measures in Parkinson disease: case study}

\author{
Fernanda Vargas Ferreira ${ }^{(1)}$, Carla Aparecida Cielo ${ }^{(2)}$, Maria Elaine Trevisan ${ }^{(3)}$
}

\begin{abstract}
RESUMO
Tema: características vocais acústicas de indivíduos com Doença de Parkinson (DP). Procedimentos: estudo de casos, estatística descritiva; cinco pares de parkinsonianos e controle, três masculinos e dois femininos, entre 36 e 63 anos. Avaliação otorrinolaringológica, fonoaudiológica, voz analisada pelo Multi-Dimensional Voice Program Advanced Model 5105 da Kay Pentax@. Resultados: a f0 das mulheres adultas de meia-idade com DP ficou na faixa masculina e a fo dos controles no limite inferior da faixa feminina. Nos demais sujeitos, que eram homens de diferentes idades, a fo foi normal; houve aumento de todas as medidas acústicas principalmente nos sujeitos com DP de todas as faixas etárias estudadas. Conclusão: o processo de envelhecimento e suas consequências parecem atuar como fator interferente nas modificações acústicas da voz, mas, aparentemente, a DP e a idade precoce de seu aparecimento podem acentuar tais alterações, repercutindo de forma negativa na fonação.
\end{abstract}

DESCRITORES: Doença de Parkinson; Voz; Acústica da fala

\section{INTRODUÇÃO}

A voz é uma das manifestações da personalidade humana, compondo a linguagem oral ${ }^{1}$; permitindo a dinâmica nas relações interpessoais ?, sendo essencial para uma comunicação efetiva. Ela é extremamente importante para que o indivíduo possa exercer as mais corriqueiras atividades de vida diária, de lazer e de trabalho, permitindo assim, a expressão de seus sentimentos e ideias ${ }^{1-3}$. A comunicação oral é complexa, pois é resultado da interação entre os sistemas nervoso central, respiratório, articulatório e fonatório. Sua produção se faz através do movimento coordenado

(1) Fisioterapeuta; Professora do Curso de Fisioterapia do Centro Universitário Franciscano, UNIFRA, Santa Maria, RS, Brasil; Mestre em Distúrbios da Comunicação Humana pela Universidade Federal de Santa Maria.

(2) Fonoaudióloga; Professora Adjunta do Departamento de Fonoaudiologia da Universidade Federal de Santa Maria, UFSM, Santa Maria, RS, Brasil; Doutora em Linguística Aplicada pela Pontifícia Católica do Rio Grande do Sul.

(3) Fisioterapeuta; Professora Assistente do Departamento de Fisioterapia e Reabilitação da Universidade Federal de Santa Maria, UFSM, Santa Maria, RS, Brasil; Mestre em Ciência do Movimento Humano - Área de Fisiologia do Exercício pela Universidade Federal de Santa Maria.

Conflito de interesses: inexistente do ar expiratório e da musculatura intrínseca da laringe, de modo que a produção da voz é oriunda de uma complexa e dinâmica interação entre diversos músculos do corpo ${ }^{1-3}$.

A partir da complexidade da produção vocal, diversas circunstâncias como a senilidade e patologias como a Doença de Parkinson (DP), podem gerar desarmonia no processo de fonação e, por conseguinte, na comunicação. O processo de enveIhecimento vocal ou presbifonia é único para cada indivíduo, sendo resultado da interação de fatores como as condições laríngeas, os aspectos psicológicos, a história de vida, fatores raciais e hereditários ${ }^{1}$. Associado à complexidade do tema, estudos apontam que não há uma idade para o início desse processo, ainda que seus efeitos acentuem-se a partir dos 55 anos para o sexo feminino, em virtude da depleção hormonal no climatério e a partir dos 60 anos para homens ${ }^{1,2,4}$. Alguns autores afirmam que vários marcadores vocais da senilidade tornam-se mais evidentes após os 60 anos de idade como as alterações da frequência fundamental, tremor vocal, instabilidade na emissão, redução da tessitura vocal e da velocidade de fala, deterioração da qualidade vocal, aumento da nasalidade, decréscimo dos tempos máximos de fonação (TMF), a intensidade vocal, e da capacidade vital (CV), menor 
uniformidade e deslocamento dos formantes, aumento de jitter e das pausas articulatórias ${ }^{1,4-6}$.

Dentre as patologias associadas à senescência, destaca-se a Doença de Parkinson (DP), cuja estimativa está em torno de 40 milhões de pessoas no mundo com diagnóstico de DP em $2020^{7}$ crescente. Contudo, no Brasil, não existem estimativas oficiais.

A DP é uma doença neurológica associada à degeneração dos gânglios basais, que são responsáveis pelo início do movimento e pela manutenção dos planos motores que o antecedem ${ }^{3,8}$. Ela é caracterizada por bradicinesia, tremor de repouso, instabilidade postural, rigidez e alterações na fala, voz e deglutição ${ }^{8,9}$.

A disartrofonia hipocinética afeta de 75 a $90 \%$ dos indivíduos com DP ${ }^{8-10}$, na qual se observa voz de intensidade reduzida, monoaltura, rouquidão, soprosidade, tremor vocal, disprosódia, imprecisão articulatória, gama tonal reduzida, pregas vocais arqueadas, fenda glótica durante a fonação e assimetria laríngea ${ }^{3,8,10}$.

Em função de a emissão vocal envolver tantos sistemas e estruturas, especialmente em doenças neurológicas, a análise acústica da voz adquire status, tanto para fins de diagnóstico quanto de prognóstico, uma vez que se associa à atuação clínica e científica ${ }^{10}$, contribuindo para a melhor compreensão desse processo. Estudo investigativo avaliou a voz de 43 parkinsonianos, de sexo feminino e sexo masculino, por meio do Multi Dimensional Voice Program Advanced, da Kay Pentax@, obtendo para as mulheres uma média de frequência fundamental (f0) de $177,37 \mathrm{~Hz}$ e para os homens, $146,05 \mathrm{~Hz}$. Em relação às medidas de perturbação da frequência, houve aumento das medidas de jitter absoluto (Jita), de jitter relativo (Jitter) e de quociente de perturbação do pitch (PPQ). Nas medidas de perturbação da amplitude, houve aumento do shimmer dB (ShdB), do shimmer relativo (Shim) e do quociente de perturbação da amplitude (APQ). Quanto às medidas de ruído, obteve-se valor elevado de NHR e VTI sem alterações; e quanto às de tremor, encontrou-se aumento na Fatr, FTRI e Fftr ${ }^{9,10}$.

O presente estudo justifica-se pela escassez e necessidade de mais estudos sobre as alterações de voz em parkinsonianos, propiciando um aprofundamento dos conhecimentos acerca dessa doença, uma vez que a DP, por dificuldades na comunicação, tende a gerar isolamento social e depressão nesses indivíduos ${ }^{3,8}$.

Dessa forma, o objetivo deste estudo é descrever as medidas acústicas da fonte glótica da voz de um grupo de homens e mulheres com DP, em comparação com casos de controle.

\section{APRESENTAÇÃO DO CASO}

O estudo caracterizou-se por uma análise descritiva, transversal e exploratória, por meio do levantamento de dados em campo.

Para a seleção dos casos de estudo, utilizaramse os critérios de inclusão: diagnóstico neurológico de DP; qualquer faixa etária, dos sexos feminino e masculino, e falantes do português brasileiro (PB).

Os critérios de exclusão foram: apresentar déficit cognitivo; apresentar avaliação laringológica com anormalidades, ou seja, alterações laríngeas típicas da DP e ou do envelhecimento; apresentar gripe, alergias respiratórias ou outra doença que no dia das avaliações; sintomas ou sinais espirométricos de doença pulmonar obstrutiva crônica ou outras patologias pulmonares; estar realizando tratamento fonoaudiológico e/ou otorrinolaringológico; ser profissional da voz, pelo provável condicionamento e preparo vocal e respiratório; ser tabagista e/ou consumir álcool em excesso (conforme a Organização Mundial da Saúde - OMS, não ingerir mais de duas doses por dia), pela agressão à laringe, podendo constituir problemas vocais orgânicos; apresentar perda auditiva, já que a audição normal é importante para o auto-monitoramento da voz e da fala; cantar em coros, a fim de evitar que o indivíduo possuísse noções de técnicas vocais. Os mesmos critérios de inclusão e de exclusão foram aplicados aos casos de controle, com exceção do diagnóstico neurológico de DP e das alterações laríngeas típicas da DP.

Após a assinatura do TCLE, todos os voluntários, com e sem DP, responderam a uma entrevista semi-estruturada e realizaram avaliação otorrinolaringológica e auditiva, por profissionais especialistas. Foram selecionados para as avaliações de coleta de dados os sujeitos que se enquadraram na pesquisa conforme os critérios de inclusão e de exclusão.

A partir da divulgação nas mídias impressa e eletrônica, apresentaram-se como voluntários oito indivíduos com DP, todos falantes do PB, sendo que cinco parkinsonianos constituíram os casos de estudo, nas idades de 36, 56, 57, 61 e 63 anos; e três foram excluídos da pesquisa por apresentarem perda auditiva, sendo encaminhados para tratamento específico. Os casos de controle constituíram-se de indivíduos sem doenças neurológicas, pareados, segundo a idade, o sexo e o nível de atividade física, com os sujeitos com DP, resultando em cinco pares. Os sujeitos foram alocados em grupos conforme a literatura ${ }^{11}$ : adulto jovem - AJ (de 20 a 40 anos), adulto de meia-idade - AMI (41 a 60 anos) e início da terceira idade - ITI (dos 61 aos 70 anos). 
A partir da formação dos casos de estudo e de controle, uma fonoaudióloga registrou a emissão sustentada da vogal /a/, em frequência e intensidade habituais, após inspiração profunda, em tempo máximo de fonação, sem uso de ar de reserva expiratória, solicitando-se ao sujeito que ficasse em pé, com os braços estendidos ao longo do corpo. O microfone acoplado ao gravador digital da marca CABO 4P BK-USB22 "BAK" foi posicionado em ângulo de $90^{\circ}$ graus da boca do sujeito, mantendose a distância de quatro $\mathrm{cm}$ entre o microfone e a boca ${ }^{1,10}$.

Para a análise acústica da voz, foram utilizados os cinco segundos iniciais da emissão da vogal /a/, sendo excluído o início da emissão para que o ataque vocal não interferisse na análise dos dados ${ }^{1,4,10}$.

As medidas, obtidas por meio do software Multi Dimensional Voice Program Advanced (MVDPA), da Kay Pentax $囚$, foram tabuladas e analisadas de forma agrupada - um grupo referente às medidas de frequência: Frequência fundamental média (f0); Frequência fundamental máxima (fhi); Frequência fundamental mínima (flo); Desvio-padrão da frequência fundamental (STD); outro grupo referente às medidas de perturbação da frequência: Jitter absoluto (Jita); Jitter percentual (Jitt); Média relativa da perturbação de frequência (RAP); Quociente de perturbação do Pitch (PPQ); Quociente de perturbação do Pitch suavizado (sPPQ); Coeficiente da variação da frequência fundamental (vf0); outro grupo referente às medidas de perturbação da amplitude: Shimmer em dB (ShdB); Shimmer percentual (Shim); Quociente de perturbação da amplitude (APQ); Quociente de perturbação da amplitude suavizado (sAPQ); Coeficiente de variação da amplitude (vAm); e um último grupo referente às medidas: Proporção ruído-harmônico (NHR); Índice de turbulência da voz (VTI); Índice de fonação suave (SPI); Índice da frequência do tremor (FTRI); Índice da amplitude do tremor (ATRI); Grau de quebra da voz (DVB); Grau dos componentes sub-harmônicos (DSH); Grau de silêncio - período sem voz (DUV); Número de quebras vocais (NVB); Número de segmentos sub-harmônicos (NSH); Número de segmentos não sonorizados (NUV). Os dados foram armazenados no banco de dados do próprio software.

Para este estudo, consideraram-se os valores de 150 a $250 \mathrm{~Hz}$ para a f0 do sexo feminino ${ }^{1} \mathrm{e}$, para os homens, a f0 entre 80 e $150 \mathrm{~Hz}{ }^{1}$. Para as demais medidas, foram considerados, para os sujeitos de cada sexo, a faixa de valores de normalidade proposta pelo programa MDVPA.

A amostra de voz foi coletada uma hora após administração da medicação (período ON).
Os dados foram analisados de forma descritiva, observando-se as características apresentadas pelos casos de estudo e de controle, com sujeitos pareados segundo idade, sexo e nível de atividade física.

O projeto de pesquisa foi previamente aprovado pelo Comitê de Ética em Pesquisa da instituição de origem (091/2005), e todos os participantes assinaram o Termo de Consentimento Livre e Esclarecido (Res. 196/96 CNS).

\section{RESULTADOS}

Os pares de casos (de estudo e de controle) foram cinco, sendo três de homens e dois de mulheres, entre 36 e 63 anos de idade, com os parkinsonianos classificados no Estágio de Hohen e Yahr Modificado ${ }^{12}$. Constituiram-se três grupos etários, conforme a literatura ${ }^{11}$ : a) grupo AJ, composto de um par de casos de 36 anos do sexo masculino, estágio 2,0 e nível de atividade física ativo; b) grupo AMI, com dois pares de casos, um de 56 , no estágio 1,5 e nível de atividade física ativo, e um de 57 anos, no estágio 2,5 e nível de atividade física irregularmente ativo $\mathrm{A}$, ambos do sexo feminino; c) grupo ITI, constituído de dois pares de casos, um de 61, no estágio 3,0 e nível de atividade física sedentário, e um de 63 anos, no estágio 2,5 e nível de atividade física muito ativo, ambos do sexo masculino (Tabela 1).

Os achados da avaliação vocal acústica dos indivíduos dos sexos feminino e masculino estão expostos nas Tabelas 1, 2, 3, 4 e 5.

\section{DISCUSSÃO}

A frequência fundamental (f0) corresponde ao número de vibrações por segundo das pregas vocais em um dado momento, produzindo no ar (meio elástico) zonas de compressão e rarefação (som) ${ }^{1,13}$. Apresenta relação direta com comprimento, tensão, rigidez e massa das pregas vocais $^{1,10,13}$, sofrendo influência da idade, do sexo e da altura do indivíduo ${ }^{1,13,14}$.

As medidas de $\mathrm{f} 0$ das parkinsonianas do grupo AMI (Tabela 2), encontraram-se inferiores à literatura, que aponta valores entre 150 e $250 \mathrm{~Hz}$ para mulheres adultas falantes do PB ${ }^{1,15}$, inclusive inferiores à média para idosas ${ }^{4}$. Ainda, a média da f0 das parkinsonianas encontrou-se na faixa masculina, concordando com os autores que apontam que na $\mathrm{DP}$, a repercussão na f0 pode ocorrer através de um decréscimo ${ }^{3,8,10,13}$. Entretanto, outros autores relatam que há aumento da frequência fundamental, o que não condiz com os resultados encontrados neste estudo de casos em relação às parkinsonia- 
Tabela 1 - Caracterização dos casos de estudo

\begin{tabular}{cccccc}
\hline $\begin{array}{c}\text { Casos de } \\
\text { estudo }\end{array}$ & Idade & Sexo & $\begin{array}{c}\text { Diagnóstico } \\
\text { da DP }\end{array}$ & $\begin{array}{c}\text { Estágio da } \\
\text { DP }\end{array}$ & $\begin{array}{c}\text { Nível de } \\
\text { atividade físico }\end{array}$ \\
\hline AJ & 36 anos & M & 6 anos & 2,0 & ativo \\
AMI & 57 anos & F & 5 anos & 2,5 & $\begin{array}{c}\text { Irregularmente } \\
\text { ativo A }\end{array}$ \\
AMI & 56 anos & F & 4 anos & 1,5 & ativo \\
ITI & 61 anos & M & 3 anos & 3,0 & sedentário \\
ITI & 63 anos & M & 5 anos & 2,5 & muito ativo \\
\hline
\end{tabular}

Legenda: adulto jovem (AJ); Doença de Parkinson (DP); adulto de meia-idade (AMI); início de terceira idade (ITI)

Tabela 2 - Resultado da análise acústica, das medidas de frequência fundamental, por meio do programa MDVPA, nos grupos

\begin{tabular}{lcccccccc}
\hline Variáveis & $\begin{array}{c}\text { Média } \\
\text { AJ } \\
\text { DP } \\
\text { masculino }\end{array}$ & $\begin{array}{c}\text { Média } \\
\text { AJ } \\
\text { masculino }\end{array}$ & $\begin{array}{c}\text { Média } \\
\text { ITI } \\
\text { DP } \\
\text { masculino }\end{array}$ & $\begin{array}{c}\text { Média } \\
\text { ITI } \\
\text { masculino }\end{array}$ & $\begin{array}{c}\text { MDVPA } \\
\text { padrão } \\
\text { masculino }\end{array}$ & $\begin{array}{c}\text { Média } \\
\text { AMI } \\
\text { DP } \\
\text { feminino }\end{array}$ & $\begin{array}{c}\text { Média } \\
\text { AMI } \\
\text { feminino }\end{array}$ & $\begin{array}{c}\text { MDVPA } \\
\text { padrão } \\
\text { feminino }\end{array}$ \\
\hline f0 $(\mathrm{Hz})$ & 148,586 & 133,925 & 119,05 & 113,11 & $<145,22^{*}$ & 138,088 & 158,316 & $145,22 a$ \\
fhi $(\mathrm{Hz})$ & 184,585 & 141,268 & 161,90 & 125,71 & 150,080 & 149,517 & 182,294 & 252,724 \\
$\mathrm{fl}_{\mathrm{O}}(\mathrm{Hz})$ & 122,262 & 126,445 & 94,45 & 104,86 & 140,418 & 104,63 & 141,58 & 234,861 \\
$\mathrm{STD}(\mathrm{Hz})$ & 6,805 & 1,744 & 5,1 & 2,85 & 1,349 & 21,13 & 4,37 & 2,722 \\
\hline
\end{tabular}

Legenda: adulto jovem (AJ); Doença de Parkinson (DP); adulto de meia-idade (AMI); início de terceira idade (ITI); frequência fundamental média (f0); frequência fundamental máxima (fhi); frequência fundamental mínima (flo); desvio-padrão da frequência fundamental (STD) *Valor não utilizado neste estudo. Consideraram-se os valores de 150 a $250 \mathrm{~Hz}$ para a f0 das mulheres ${ }^{1}$, para os homens, a f0 entre 80 e $150 \mathrm{~Hz}^{1}$

Tabela 3 - Resultado da análise acústica, das medidas de perturbação da frequência, por meio do programa MDVPA, nos grupos

\begin{tabular}{|c|c|c|c|c|c|c|c|c|}
\hline Variáveis & $\begin{array}{c}\text { Média } \\
\text { AJ } \\
\text { DP } \\
\text { masculino }\end{array}$ & $\begin{array}{c}\text { Média } \\
\text { AJ } \\
\text { masculino }\end{array}$ & $\begin{array}{c}\text { Média } \\
\text { ITI } \\
\text { DP } \\
\text { masculino }\end{array}$ & $\begin{array}{c}\text { Média } \\
\text { ITI } \\
\text { masculino }\end{array}$ & $\begin{array}{c}\text { MDVPA } \\
\text { padrão } \\
\text { masculino }\end{array}$ & $\begin{array}{c}\text { Média } \\
\text { AMI } \\
\text { DP } \\
\text { feminino }\end{array}$ & $\begin{array}{c}\text { Média } \\
\text { AMI } \\
\text { feminino }\end{array}$ & $\begin{array}{c}\text { MDVPA } \\
\text { padrão } \\
\text { feminino }\end{array}$ \\
\hline Jita $\mu \mathrm{s}$ & 141,567 & 85,364 & 285,7 & 204,4 & 83,20 & 92,2 & 72,58 & 83,20 \\
\hline Jitter \% & 2,099 & 1,143 & 3,16 & 2,23 & 1,040 & 1,19 & 1,15 & 1,040 \\
\hline RAP $\%$ & 1,204 & 0,672 & 1,97 & 1,40 & 0,680 & 0,73 & 0,70 & 0,680 \\
\hline PPQ \% & 1,251 & 0,689 & 1,54 & 1,24 & 0,840 & 0,83 & 0,74 & 0,840 \\
\hline sPPQ \% & 1,812 & 0,848 & 2,02 & 1,73 & 1,020 & 4,80 & 0,83 & 1,220 \\
\hline vf0 \% & 4,580 & 1,302 & 4,36 & 2,65 & 1,100 & 16,05 & 2,76 & 1,100 \\
\hline
\end{tabular}

Legenda: adulto jovem (AJ); Doença de Parkinson (DP); adulto de meia-idade (AMI); início de terceira idade (ITI); Jitter absoluto (Jita); Jitter percentual (Jitt); Média relativa da perturbação da frequência (RAP); Quociente de perturbação do Pitch (PPQ); Quociente de perturbação do Pitch suavizado (sPPQ); Coeficiente da variação da frequência fundamental (vfO)

nas AMI ${ }^{15-17}$. Essa discrepância pode decorrer das flutuações associadas ao uso da levodopa (período $\mathrm{ON}$ ), que tende a variar a frequência fundamental, no entanto, sua influência sobre a qualidade vocal é inconclusiva, já que, achados neuroquímicos sugerem que vias não-dopaminérgicas atuem sobre os distúrbios da voz e da fala na DP ${ }^{9,16-18}$.

Os casos de controle das parkinsonianas, também do grupo AMI, encontraram-se dentro da faixa de normalidade, conforme a literatura ${ }^{1,15}$ (Tabela 2), porém no limiar inferior. É possível que a interferência do processo de envelhecimento vocal não tenha repercutido de maneira significativa sobre a f0, uma vez que compromete mais alguns indivíduos em relação a outros e resulta de fatores intrínsecos e extrínsecos ${ }^{9}$, contudo, a f0 no limiar inferior da faixa feminina pode estar relacionada ao decréscimo de hormônios sexuais gerado pelo processo do 
Tabela 4 - Resultado da análise acústica, das medidas de perturbação da amplitude, por meio do programa MDVPA, nos grupos

\begin{tabular}{|c|c|c|c|c|c|c|c|c|}
\hline Variáveis & $\begin{array}{c}\text { Média } \\
\text { AJ } \\
\text { DP } \\
\text { masculino }\end{array}$ & $\begin{array}{c}\text { Média } \\
\text { AJ } \\
\text { masculino }\end{array}$ & $\begin{array}{c}\text { Média } \\
\text { ITI } \\
\text { DP } \\
\text { masculino }\end{array}$ & $\begin{array}{c}\text { Média } \\
\text { ITI } \\
\text { masculino }\end{array}$ & $\begin{array}{c}\text { MDVPA } \\
\text { padrão } \\
\text { masculino }\end{array}$ & $\begin{array}{c}\text { Média } \\
\text { AMI } \\
\text { DP } \\
\text { feminino }\end{array}$ & $\begin{array}{c}\text { Média } \\
\text { AMI } \\
\text { feminino }\end{array}$ & $\begin{array}{c}\text { MDVPA } \\
\text { padrão } \\
\text { feminino }\end{array}$ \\
\hline ShdB (dB) & 0,862 & 0,755 & 0,81 & 1,13 & 0,350 & 0,77 & 0,54 & 0,350 \\
\hline Shim \% & 10,240 & 8,385 & 8,63 & 12,78 & 3,810 & 8,82 & 6,34 & 3,810 \\
\hline APQ \% & 7,820 & 6,618 & 6,98 & 9,50 & 3,070 & 6,07 & 4,81 & 3,070 \\
\hline sAPQ \% & 14,158 & 8,268 & 10,43 & 13,17 & 4,230 & 7,93 & 9,39 & 4,230 \\
\hline vAm \% & 37,812 & 19,346 & 27,46 & 17,89 & 8,200 & 16,61 & 21,42 & 8,200 \\
\hline
\end{tabular}

Legenda: adulto jovem (AJ); Doença de Parkinson (DP); adulto de meia-idade (AMI); início de terceira idade (ITI); Shimmer em dB (ShdB); Shimmer percentual (Shim); Quociente de perturbação da amplitude (APQ); Quociente de perturbação da amplitude suavizado (SAPQ); Coeficiente de variação da amplitude (vAm)

Tabela 5 - Resultado da análise acústica, das medidas de ruído, de quebra de voz, de segmentos não sonorizados, de sub-harmônicos e de tremor, por meio do programa MDVPA, nos grupos

\begin{tabular}{|c|c|c|c|c|c|c|c|c|}
\hline Variáveis & $\begin{array}{c}\text { Média } \\
\text { AJ } \\
\text { DP } \\
\text { masculino }\end{array}$ & $\begin{array}{c}\text { Média } \\
\text { AJ } \\
\text { masculino }\end{array}$ & $\begin{array}{c}\text { Média } \\
\text { ITI } \\
\text { DP } \\
\text { masculino }\end{array}$ & $\begin{array}{c}\text { Média } \\
\text { ITI } \\
\text { masculino }\end{array}$ & $\begin{array}{c}\text { MDVPA } \\
\text { padrão } \\
\text { masculino }\end{array}$ & $\begin{array}{c}\text { Média } \\
\text { AMI } \\
\text { DP } \\
\text { feminino }\end{array}$ & $\begin{array}{c}\text { Média } \\
\text { AMI } \\
\text { feminino }\end{array}$ & $\begin{array}{c}\text { MDVPA } \\
\text { padrão } \\
\text { feminino }\end{array}$ \\
\hline $\mathrm{NHR}$ & 0,189 & 0,177 & 0,307 & 0,22 & 0,190 & 0,182 & 0,151 & 0,190 \\
\hline VTI & 0,055 & 0,041 & 0,060 & 0,050 & 0,061 & 0,069 & 0,059 & 0,061 \\
\hline SPI & 7,019 & 11,192 & 12,292 & 12,22 & 14,120 & 5,1 & 6,80 & 14,120 \\
\hline DVB & 0 & 0 & 5,97 & 0,81 & 1,000 & 0 & 0 & 1,000 \\
\hline NVB & 0 & 0 & 2 & 0,5 & 0,900 & 0 & 0 & 0,900 \\
\hline DUV & 16,949 & 2,055 & 25,85 & 18,79 & 1,000 & 7,14 & 0 & 1,000 \\
\hline NUV & 10 & 3 & 38,5 & 13,5 & 0,900 & 6,5 & 0 & 0,900 \\
\hline $\mathrm{DSH}$ & 0 & 0 & 0 & 0 & 1,000 & 7,31 & 0 & 1,000 \\
\hline $\mathrm{NSH}$ & 0 & 0 & 0 & 0 & 0,900 & 6,5 & 0 & 0,900 \\
\hline FTRI & 1,711 & 0,376 & 1,191 & 0,81 & 0,950 & 0,21 & 0,25 & 0,950 \\
\hline ATRI & 14,055 & 10,406 & 1,72 & 0,30 & 4,370 & 5,05 & 7,95 & 4,370 \\
\hline
\end{tabular}

Legenda: adulto jovem (AJ); Doença de Parkinson (DP); adulto de meia-idade (AMI); início de terceira idade (ITI); Proporção ruídoharmônico (NHR); Índice de turbulência da voz (VTI); Índice de fonação suave (SPI); Índice da frequência do tremor (FTRI); Índice da amplitude do tremor (ATRI); Grau de quebra da voz (DVB); Grau dos componentes sub-harmônicos (DSH); Grau de silêncio - período sem voz (DUV); Número de quebras vocais (NVB); Número de segmentos sub-harmônicos (NSH); Número de segmentos não sonorizados (NUV)

envelhecimento, que torna a voz mais grave e com tessitura vocal mais restrita $2,4-6$.

Ainda em relação ao grupo $\mathrm{AMI}$, verificou-se que os casos de estudo apresentaram STD acima do esperado e também superiores à dos seus casos de controle, sugerindo instabilidade fonatória, característica comum na DP ${ }^{3,9}$. Os casos de controle AMI apresentaram STD acima do esperado (mas bem abaixo dos casos de estudo), podendo-se considerar como variações aceitáveis na fonação de indivíduos normais ${ }^{1,10}$ ou, ainda, devidas ao início do processo de envelhecimento, com suas características de instabilidade na emissão, comuns a partir

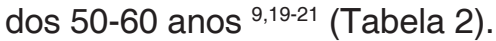

O parkinsoniano AJ apresentou fo na faixa esperada para adultos do sexo masculino, sendo considerada normal, contudo, próximo ao limiar inferior do sexo feminino ${ }^{1,15}$ (Tabela 2). Existem estudos que apontam uma tendência de elevação da fo possivelmente, pela rigidez da musculatura laríngea e respiratória, que contribuiria para a elevação da fo, sendo esta proporcional ao estágio da doença 3,16,17. Além disso, a elevação da f0 poderia ser possível uma vez que, no período ON, em decorrência dos fármacos derivados da levodopa, pode ocorrer melhora discreta das medidas acústicas ${ }^{10,17}$. No entanto, não há consenso na literatura, uma vez que outros autores afirmam que as desordens da fala e da voz apresentam pouca responsividade à medicação ${ }^{16,18}$. Esses achados estão de acordo com o estudo cuja análise vocal foi obtida através do CSL 4300, com média de f0 de $180,1 \mathrm{~Hz}$ para indivíduos com DP do sexo masculino ${ }^{22}$, também convergem com os resultados do trabalho que, 
através do CSL, nas fases ON e OFF, encontrou média de fo de 150,8 Hz para os homens ${ }^{8}$, e, ainda, com os pesquisadores que, por meio do GRAM, exibiram média de f0 entre os homens de $146,3 \mathrm{~Hz}{ }^{17}$. O caso de controle AJ também apresentou f0 dentro do esperado pela literatura ${ }^{1,15}$ (Tabela 2).

Ainda em relação ao par de AJ, verificou-se que o parkinsoniano apresentou medidas de fhi e STD acima do esperado e também superiores às do seu caso de controle, possivelmente, sinalizando instabilidade fonatória, achado comum na DP ${ }^{3,9}$. O caso de controle AJ apresentou STD acima do esperado (mas bem abaixo do caso de estudo), sendo que são consideradas aceitáveis variações na emissão vocal de indivíduos normais, em virtude da influência de fatores individuais ${ }^{1,10}$. O mesmo ocorreu com o grupo ITI em relação aos casos de estudo e de controle, evidenciando maior influência negativa da DP do que do processo de envelhecimento nesses casos, uma vez que características como qualidade vocal alterada, tremor, tessitura vocal reduzida e instabilidade na emissão, que são comuns a partir dos 50-60 anos 4,6,9, tendem a ser exacerbadas com o avanço da idade e podem ser intensificadas pela DP ${ }^{3,16}$ (Tabela 2).

Os achados do AJ com DP podem ser justificados conforme o "parkinsonismo de início precoce", que acomete indivíduos com idade inferior a 40 anos e tende a gerar sintomatologia mais grave, inclusive na disartrofonia hipocinética ${ }^{10,18,20,21}$. Acredita-se que o principal fator de interferência nos valores elevados das medidas vocais acústicas do caso de estudo AJ tenha sido a doença ou a deterioração do sistema nervoso central, visto que a laringe ainda não apresenta as modificações decorrentes do processo de envelhecimento.

Ainda, os homens do grupo ITI, com e sem DP (Tabela 2), apresentaram f0 dentro do esperado para a faixa etária do sexo masculino ${ }^{1,15}$. Poderse-ia esperar a elevação da f0 pelas modificações decorrentes da DP, no grupo de estudo, devido à rigidez da musculatura laríngea e respiratória ${ }^{3,16,17}$ e, no grupo de controle, uma vez que, no processo de envelhecimento masculino, a literatura aponta a elevação da f0, o que não ocorreu nesses casos ${ }^{2,5,6}$ (Tabela 2).

As medidas de perturbação ciclo-a-ciclo avaliam as variações do sinal acústico, sendo referentes a um determinado período de vibração glótica, com relação à frequência (Jitter) e à intensidade (Shimmer) ${ }^{1,10,13}$.

No que se refere aos valores de perturbação da frequência (medidas de jitter) (Tabela 3), observouse no AJ com DP que todas as medidas apresentaram-se elevadas tanto em relação ao esperado quanto em relação ao seu controle, sugerindo instabilidade do ajuste fonatório, resultante de redução no controle motor e de disfonia ${ }^{9,10}$. Achados similares foram encontrados em outros estudos ${ }^{23,24}$. Contudo, ao se considerar a voz adulta jovem, a tendência é de estabilidade e com características próprias de cada sexo ${ }^{1,4,14}$, ou seja, tais alterações no parkinsoniano jovem presumivelmente denotam um impacto negativo advindo da DP que gera instabilidade na emissão vocal $3,10,16-18,20,21,25$. O seu controle apresentou apenas três das seis medidas de jitter elevadas, mas próximas do limiar de normalidade, o que pode ser justificado pela possível influência de fatores sobre a produção vocal, como as características estruturais das pregas vocais como a forma, o tamanho, a densidade e a tensão, bem como as emoções ${ }^{14}$.

Todos os sujeitos com DP dos grupos AMI e ITI e todos os controles do grupo ITI apresentaram todas as medidas de jitter elevadas, sendo que os casos com DP ainda as apresentaram acima dos casos controle. Nos casos de controle do grupo AMI, apenas duas medidas de jitter ficaram acima do esperado. Estudos citam o aumento de medidas de jitter como característica da disartrofonia hipocinética ${ }^{6,9,26}$, da inabilidade em sustentar a emissão devido à DP $3,9,10,16$ bem como do processo de enveIhecimento vocal ${ }^{4-6}$. Pesquisas mostram aumento de jitter em adultos de meia-idade, especialmente a partir dos 60 anos, resultante da redução da mobilidade laríngea e da menor eficiência biomecânica $2,4,5$, uma vez que modificações na laringe iniciam na terceira idade, acentuando-se a partir dos 65 - 70 anos, ilustradas pela atrofia progressiva da musculatura e da mucosa das pregas vocais, pelo afrouxamento de ligamentos e também por alteração no controle neurofisiológico, podendo gerar instabilidade ${ }^{4-6}$. Esse aumento, porém, não necessariamente denota lesão estrutural nas pregas vocais e sim, pode sinalizar dificuldades de controle do sistema fonatório e/ou respiratório, uma vez que tais medidas fornecem indícios da irregularidade da vibração da mucosa das pregas vocais, como variação de massa, de tensão e distribuição do muco, de simetria das estruturas vocais, bem como da relação entre as características biomecânicas e de controle neuromuscular ${ }^{1,13}$ (Tabela 3).

Dessa forma, pode-se supor que valores elevados de jitter, indicativos de instabilidade na oscilação das pregas vocais, cuja qualidade vocal pode se apresentar rouca ${ }^{1,9,17}$, estão presentes tanto no processo de envelhecimento vocal quanto na DP. Em estudo de análise acústica com 12 parkinsonianos nos períodos ON e OFF, apontou altos valores de jitter em ambas as fases ${ }^{27}$. Similarmente, 
em outro trabalho com parkinsonianos na fase ON, obteve-se elevados valores de jitter ${ }^{26}$.

Em relação às medidas de shimmer (Tabela 4), todos os sujeitos da pesquisa, tanto os casos de estudo como os de controle, as apresentaram elevadas, salientando-se que o caso de estudo AJ as apresentou maiores ainda do que seu caso de controle, resultados em consonância com a literatura ${ }^{9,19,26}$ (Tabela 4). O shimmer reflete a perturbação da amplitude ciclo-a-ciclo, bem como a diminuição ou inconsistência do coeficiente de contato das pregas vocais, podendo relacionar-se à presença de soprosidade na voz ou ao ruído como um todo $1,8,9,13,19$. Tais características são comuns no processo de envelhecimento, sendo que a deterioração da voz com a idade ocorre em grau variável ${ }^{1,2,4-6,13}$, e provavelmente sejam exacerbadas pela DP ${ }^{3,28}$ atuando como fator negativo associado, com piora das medidas acústicas devido à instabilidade na emissão, decorrente de alterações neuromusculares e funcionais ${ }^{1,3,4,22}$.

Além disso, verificou-se que, em laringes normais, o shimmer absoluto mostrou-se maior para o sexo masculino, embora nem sempre com diferença estatisticamente significante ${ }^{14} \mathrm{e}$ que as medidas de shimmer tendem a aumentar com o envelhecimento nos homens ${ }^{4-6}$.

Tais achados, por fim, podem também sugerir incoordenação pneumofonoarticulatória (IPFA) ${ }^{1,8,13}$, visto que tende a haver uma assincronia entre os movimentos tóraco-abdominais e laríngeos ${ }^{8,20,21}$, por fraqueza muscular respiratória, limitações músculo-esqueléticas da coluna vertebral e envolvimento das vias aéreas superiores ao nível de estruturas supra e infra-glóticas ${ }^{4,6,13}$. Entretanto, é possível que na DP haja maior repercussão, pois a rigidez e a bradicinesia podem comprometer de forma mais acentuada a resposta contrátil e, consequentemente, sua funcionalidade 4,9,17,22,27.

Observou-se que todos os sujeitos do grupo ITI apresentaram NHR elevado (Tabela 5), que indica a presença de ruído no sinal glótico analisado, incluindo variações da amplitude e frequência, ruído de turbulência, componentes sub-harmônicos e/ou quebras de voz ${ }^{1,10,13,14}$. Ainda, contrasta o sinal regular das pregas vocais com o sinal irregular das pregas vocais e do trato vocal, oferecendo um índice que relaciona o componente harmônico versus o componente de ruído da onda acústica ${ }^{1}$, sendo que os parkinsonianos apresentaram os maiores valores, o que denota fonação aperiódica, podendo ser reflexo de que o processo de envelhecimento compromete a qualidade da produção vocal, sendo mais evidente no sexo masculino, especialmente a partir da sexta década de vida ${ }^{4-6} \mathrm{e}$ que pode ser agravado pela presença de DP, como evidenciado em diversos estudos que encontraram valores de NHR elevados em parkinsonianos 21,26,29.

Em todos os grupos estudados, a medida de SPI ou o índice de fonação suave ficou dentro do esperado. O SPI mede o nível de energia média entre as baixas e as altas frequências, relacionando-se à completude de fechamento glótico e podendo sinalizar escape aéreo à fonação, quando elevado ${ }^{10,14}$. No entanto, pacientes com tensão podem ter SPI normal e pacientes sem desordem vocal podem ter SPI elevado ${ }^{10}$. O VTI, medida similar ao SPI, que indica o ruído de turbulência na emissão vocal devido a alterações na coaptação glótica, relacionando-se ao escape de ar à fonação ${ }^{9,10,13,14}$, também apresentou-se normal em todos os sujeitos, exceto nos DP do grupo AMI que mostraram VTI de 0,069 contra 0,061 da normalidade. Assim, os resultados obtidos estão de acordo com a literatura, uma vez o SPI e o VTI não são medidas que possam ser correlacionadas positivamente com algum tipo de alteração vocal específica, nem interpretadas isoladamente ${ }^{10,14}$ (Tabela 5).

Os parâmetros DVB e NVB, que mensuram o grau e o número de quebras vocais da emissão, correlacionando-se com períodos não-harmônicos e irregularidades na emissão vocal ${ }^{9,10,14}$, apresentaram-se elevados apenas nos DP do grupo ITI, o que pode sugerir a irregularidade da emissão relacionada ao processo de envelhecimento ${ }^{4}$, mas acentuada pela DP ${ }^{10}$, o que converge para os resultados de NHR deste grupo discutidos anteriormente (Tabela 5).

As medidas DUV e NUV que significam grau e número de segmentos não-sonorizados onde não se pode detectar a fo ${ }^{9,10,14}$ mostraram-se acima da normalidade em todos os sujeitos dos três grupos estudados, com exceção dos casos de controle do grupo AMI, podendo indicar irregularidade ou incoordenação na vocalização, desarmonia na emissão vocal com piora da qualidade vocal ${ }^{26,29}$, aperiodicidade de vibração com rouquidão e ou aspereza ${ }^{1,9,10}$ (Tabela 5).

As medidas de DSH e NSH, que representam o grau e o número de componentes sub-harmônicos, podendo corresponder a vozes diplofônicas ou emissões em som basal 9,10,14, quando elevadas ${ }^{14}$, ficaram acima da normalidade nos DP do grupo AMI, de acordo com a literatura sobre a disartrofonia hipocinética ${ }^{9,10}$ e sobre o processo de envelhecimento de mulheres, quando passam a utilizar emissões em fry ${ }^{4,14}$.

O tremor vocal, relacionado tanto a patologias neurológicas quanto ao processo de enveIhecimento, consiste na oscilação rítmica e involuntária da voz, com alterações no pitch e na 
loudness, durante a fonação ${ }^{1,5,6}$, sendo relacionado à frequência (FTRI) e à intensidade (ATRI) 1,5,6,10,14.

Todos os DP de todos os grupos etários estudados apresentaram medidas de FTRI e ATRI elevadas. Ainda, os casos de controle dos grupos AJ e AMI apresentaram as medidas de ATRI elevadas.

Tais alterações sugerem instabilidade fonatória associada a oscilações no sistema abdutor-adutor laríngeo, no músculo cricotireóideo ou nas pregas vestibulares ${ }^{1,9,10}$. A impressão e ou presença do tremor na voz indica menor capacidade de sustentação da f0 ${ }^{1,4,9,10,13}$. Autores sinalizam que o tremor é uma característica do processo de envelhecimento vocal ${ }^{1,4-6}$, bem como outros apontam o tremor também como característica da DP ${ }^{3,10,18,25,26}$, o que justifica tais achados tanto nos casos de estudo quanto nos de controle, com exceção do AJ que pode ter apresentado ATRI elevada como sinal de incoordenação pneumofonoarticulatória, uma vez que o tremor pode ocorrer não apenas por distúrbios neurológicos, mas também por fatores interferentes como ansiedade, estresse, emoção, idade e frio que podem gerar instabilidade fonatória ${ }^{1}$, e ainda pode ser considerado fisiológico, sendo esperado na emissão vocal normal ${ }^{10}$.

Com base nos resultados obtidos e em sua discussão com a literatura, notou-se que, a partir dos reflexos do processo de envelhecimento vocal, cujas repercussões ocorrem por meio da associação entre modificações anatômicas e fisiológicas, dentre elas os distúrbios no suporte respiratório, atrofia nos tecidos musculares e neurais, calcificação das cartilagens, incluindo as laríngeas, e perda de fibras elásticas e colágenas nas pregas vocais $^{1,4-6}$, é possível que a DP possa exacerbar os déficits advindos com o avanço da idade e, por consequência, dificultar a distinção entre o fisiológico e o patológico, gerando maior deterioração vocal.
Os achados encontrados nesta pesquisa são restritos à população pesquisada, na qual o controle refere-se ao pareamento de casos-controle. Desta forma, impedem-se generalizações. As diferenças entre as medidas foram discutidas de modo descritivo, ou seja, as medidas elevadas não necessariamente são valores representativos da magnitude da alteração.

Esta discussão levanta hipóteses, destacandose que são necessárias pesquisas mais aprofundadas envolvendo análises vocais complementares perceptivas e visuais, além das acústicas ${ }^{30}$.

E por fim, deve-se salientar que a análise acústica da voz ainda não oferece medidas que apresentem correlação positiva forte com determinado aspecto anátomo-fisiológico ou perceptivo-auditivo da produção vocal e que, desta forma, seus resultados devem ser utilizados como indicadores complementares das demais avaliações fonoaudiológicas e médicas.

\section{CONCLUSÃO}

Neste estudo de casos, pôde-se concluir que a f0 das mulheres AMI com DP se apresentou na faixa masculina e os casos de controle no limite inferior da faixa de normalidade feminina. Nos demais sujeitos, que eram homens de diferentes idades, a fo se apresentou na faixa de normalidade.

$\mathrm{O}$ aumento de todas as medidas acústicas principalmente nos sujeitos com DP de todas as faixas etárias estudadas sugere que é possível que o processo de envelhecimento e suas consequências atuem como fator interferente nas medidas acústicas da voz, mas, aparentemente, a DP e a idade precoce de seu aparecimento podem acentuar tais alterações, repercutindo de forma negativa na fonação.

\section{ABSTRACT \\ Background: vocal acoustic measures in individuals with Parkinson Disease (PD). Procedures: case studies, descriptive statistics; five pairs of Parkinson patients and control group, three male and two female subjects, between 36 and 63-year old. Evaluations on otorhinolaryngology, speech therapy, voice analyzed by the Multi-Dimensional Voice Program Advanced Model 5105 by Kay Pentax®. Results: f0 in women adults of middle age with PD, was in full male and fO of the controls on the bottom of the band of women. In other subjects, who were men of different ages, f0 was normal; there was an increase for all acoustic measures, mainly, in the subjects with PD of all studied age groups. Conclusion: aging process and its consequences seem to act as an interference factor in the changes of acoustic voice, but apparently, PD and the early age of its appearance may come to enhance these changes, reflecting a negative impact on phonation.}

KEYWORDS: Parkinson Disease; Voice; Speech Acoustics 


\section{REFERÊNCIAS}

1.Behlau M. Voz: o livro do especialista. vol. 1. Rio de Janeiro: Revinter, 2001.

2. Mifune E, Justino VSS, Camargo Z, Gregio F. Análise acústica da voz do idoso: caracterização da freqüência fundamental. Rev. CEFAC. 2007; 9(2):238-47. http://dx.doi.org/10.1590/ S1516-18462007000200013

3. Rosa JC, Cielo CA, Cechella C. Função fonatória em pacientes com Doença de Parkinson: uso de instrumento de sopro. Rev. CEFAC. 2009; 11(2):305-13. http://dx.doi.org/10.1590/ S1516-18462009000200016

4. Behlau M. Presbifonia: envelhecimento vocal inerente à idade. In: Russo, IP. Intervenção fonoaudiológica na terceira Idade. São Paulo: Revinter; 1999. p. 25-50.

5. Rocha TF, Amaral FP, Hanayama EM. Extensão vocal de idosos coralistas e não coralistas. Rev. CEFAC. 2007; 9(2):248-54. http://dx.doi. org/10.1590/S1516-18462007000200014

6. Feijó A, Estrela F, Scalco M. Avaliação perceptiva e quantitativa da voz na terceira idade. Rev Fonoaudiol Bras. 1998; 1(1):21-9.

7. Dias AE, Barbosa ER, Coracini K, Maia F, Marcolin MA,FregniF. Effects ofrepetitivetranscranialmagnetic stimulation on voice and speech in Parkinson's disease. Acta Neurol Scand. 2006; 113(2):92-9. http://10.1111/j.1600-0404.2005.00558.x

8. Carrara-de Angelis E. Deglutição, configuração laríngea, análise clínica e análise computadorizada da voz de pacientes com doença de Parkinson. [tese] São Paulo (SP): Universidade Federal de São Paulo; 2000. 144f.

9. Cervantes O. Doenças neurológicas (repercussões laríngeas e vocais). In: Dedivitis RA, Barros APB. Métodos de avaliação e diagnóstico de laringe e voz. São Paulo: Lovise; 2002. p. 145-75.

10. Barros APB, Carrara-deAngelis E. Análise acústica da voz. In: Dedivitis RA, Barros APB. Métodos de avaliação e diagnóstico de laringe e voz. São Paulo: Lovise; 2002.p.201-21.

11. Gallahue DL, Ozmun JC. Compreendendo o desenvolvimento motor: bebês, crianças, adolescentes e adultos. São Paulo: Phorte; 2003.

12. Hoehn MM, Yahr MD. Parkinsonism: onset, progression and mortality. Neurol. 1967; 17(5):427-42. 13. Wertzner HF, Schreiber S, Amaro L. Análise da freqüência fundamental, jitter, shimmer e intensidade vocal em crianças com transtorno fonológico. Rev Bras Otorrinolaringol. 2005; 71(5):582-8.

14. Costa Beber B. Características vocais acústicas de homens com voz e laringe normais. [dissertação] Santa Maria (RS): Universidade Federal de Santa Maria; 2009. 100f.
15. Felippe ACN, Grillo MHMM, Grechi TH. Normatização de medidas acústicas para vozes normais. Rev Bras Otorrinolaringol. 2006; 72(5):659-64.

16. D'Alatri L, Paludetti G, Contarino MF, Galla S, Marchese MR, Bentivoglio AR. Effects of bilateral subthalamic nucleus stimulation and medication on parkinsonian speech impairment. J Voice. 2008; 22(3):365-72.

17. Carrillo L, Ortiz KZ. Análise vocal (auditiva e acústica) nas disartrias. Pró-Fono. 2007; 19(4):381-6.

18. Azevedo LL, Cardoso F. Ação da levodopa e sua influência na voz e na fala de indivíduos com doença de Parkinson: [revisão]. Rev Soc Bras Fonoaudiol. 2009; 14(1):136-41.

19. Spencer KA, Rogers MA. Speech motor programming in hypokinetic and ataxic dysarthria. Brain Lang. 2005; 94(3):347-66.

20. Mourão LF, Aguiar PMC, Ferraz FAP, Behlau MS, Ferraz HB. Acoustic voice assessment in Parkinson's disease patients submitted to posteroventral pallidotomy. Arq Neuropsiquiatr. 2005; 63(1):20-5.

21. Rosen KM, Kent RD, Delaney AL, Duffy JR. Parametric quantitative acoustic analysis of conversation produced by speakers with dysarthria and healthy speakers. J Speech Lang Hear Res. 2006; 49(2):395-411.

22. Gamboa J, Jiménez-Jiménez FJ, Mate MA, Cobeta I. Alteraciones de la voz causadas por enfermedades neurológicas. Rev. Neurol. 2001; 33(2):153-68.

23. Quedas A, Duprat AC, Gasparini G. Implicações do efeito Lombard sobre a intensidade, freqüência fundamental e estabilidade da voz de indivíduos com doença de Parkinson. Rev Bras Otorrinolaringol. 2007; 73(5):675-83.

24. Goberman AM. Correlation between acoustic speech characteristics and non-speech motor performance in Parkinson Disease. Med Sci Monit. 2005; 11(3):CR109-16.

25. Morris ME. Movement disorders in people with Parkinson disease: a model for physical therapy. Phys Ther. 2000; 80(6):578-97.

26. Ramig LA, Scherer RC, Titze IR, Ringel $\mathrm{SP}$. Acoustic analysis of voices of patients with neurologic disease: rationale and preliminary data. Ann Otol Rhinol Laryngol. 1988; 97(2Pt1):164-72.

27. Dromey C. Spectral measures and perceptual ratings of hypokinetic dysarthria. J Med Speech Lang Pathol. 2003; 11(2):85-94.

28. Ferreira FV, Prado ALC, Cielo CA, Busanello AR. A relação da postura corporal com a prosódia na doença de parkinson: estudo de caso. Rev. CEFAC. 2007; 9(3):319-29. http://dx.doi.org/10.1590/ S1516-18462007000300005 
29. Rahn III DA, Chou M, Jiang JJ, Zhang Y. Phonatory impairment in Parkinson's disease: evidence from nonlinear dynamic analysis and perturbation analysis. J Voice. 2007; 21(1):64-71.
30. Gama ACC, Behlau M. Estudo da constância de medidas acústicas de vogais prolongadas e consecutivas em mulheres sem queixa de voz e em mulheres com disfonia. Rev Soc Bras Fonoaudiol. 2009; 14(1):8-14.

DOI: 10.1590/S1516-18462010005000020

RECEBIDO EM: 16/01/2009

ACEITO EM: 28/11/2009

Endereço para correspondência:

Fernanda Vargas Ferreira

Rua Visconde de Pelotas, 517

Santa Maria - RS

CEP: 97010-440

E-mail: nandaf_pg@yahoo.com.br 